\title{
Prevalencia de Giardiasis y Parásitos Intestinales en Preescolares de Hogares atendidos en un programa estatal en Armenia, Colombia
}

\author{
Jorge M. Giraldo-Gómez ${ }^{1}$ Fabiana Lora ${ }^{2}$, Luz H. Henao ${ }^{3}$, Shirley Mejía ${ }^{4}$ y Jorge \\ E. Gómez-Marín \\ ${ }^{1}$ Licenciado en Biología. Grupo de Estudio en Parasitología Molecular (GEPAMOL). Centro de \\ Investigaciones Biomédicas. Universidad del Quindío. Av. Bolívar 12N, Armenia (Quindío), \\ Colombia, Sur América. Tel/Fax +57 67 460168. Armenia- Colombia. \\ E-mail: gamaloque@yahoo.es \\ ${ }^{2}$ Licenciada en Biología. Grupo de Estudio en Parasitología Molecular (GEPAMOL). Centro de \\ Investigaciones Biomédicas. Universidad del Quindío. Av. Bolívar 12N, Armenia (Quindío), \\ Colombia, Sur América. Tel/Fax +57 67 460168. Armenia- Colombia. \\ E-mail: fabisuarez6@hotmail.com. \\ ${ }^{3}$ Bacterióloga. Red Salud Armenia. Guaduales de la Villa. Circasia, Quindio. \\ ${ }^{4}$ Nutricionista Dietitsta. Docente Programa de Medicina. Facultad Ciencias de la Salud. . \\ Universidad del Quindío. Av. Bolívar 12N, Armenia (Quindío), Colombia, Sur América. Tel/Fax \\ +57 67 460129. Armenia- Colombia. E-mail: shilito@hotmail.com \\ ${ }^{5}$ Médico. MSc. Biomedicina Tropical PhD. Biología Parasitaria, Post-doctorado Espectroscopía \\ Biomolecular. Director Grupo de Estudio en Parasitología Molecular (GEPAMOL). Centro de \\ Investigaciones Biomédicas. Universidad del Quindío. Av. Bolívar 12N, Armenia (Quindío), \\ Colombia, Sur América. Tel/Fax +57 67460149 Armenia- Colombia. \\ Email: gepamol2@uniquindio.edu.co
}

Recibido 2 Diciembre 2004/Enviado para Modificación 8 Mayo 2005/Aceptado 29 Agosto 2005

\section{RESUMEN}

Objetivo El propósito de este estudio es el de obtener datos sobre la giardiasis y el parasitismo intestinal en una muestra significativa de los niños en edades pre-escolares de los hogares de madres comunitarias del Instituto Colombiano de Bienestar Familiar (ICBF).

Materiales y Métodos Se recolectó un seriado de tres muestras de heces en 328 niños, con previo consentimiento del tutor legal. Se le realizó el análisis de laboratorio por examen directo microscópico y macroscópico utilizando Lugol al $1 \%$, solución salina al 0,83 \%, solución salina-eosina, Kato Katz y formol-éter o metodo de concentración de Ritchie (prueba comprobatoria). Las muestras positivas se conservaron en formol salino.

Resultados LoS parásitos con potencial patógeno tuvieron las siguientes prevalencias: Ascaris lumbricoides 2,4\%, Hymenolepsis nana 0,6 \%, Trichuris trichura 2,1 \%, Blastocystis hominis 6,1\% y Giardia lamblia $13 \%$. 
Conclusiones Este estudio muestra la baja frecuencia de los helmintos con respecto a los protozoos, por lo tanto estrategias de desparasitación masiva con dosis únicas de Albendazol no tienen utilidad. Se recomienda la vigilancia periódica de parásitos por exámenes en estos niños. Se confirma que el parásito patógeno mas prevalente es Giardia lamblia en los niños de las guarderias infantiles del ICBF. La prevalencia de Giardia no se relacionó de manera estadisticamente significativa con el estado nutricional de los niños de las guarderias del ICBF. La mayoria de niños infectados por Giardia son asintomáticos.

Palabras Clave: Giardiasis, prevalencia, nutrición del niño, preescolar, parásitos (fuente: DeCS, BIREME)

ABSTRACT

Prevalence of giardiasis and intestinal parasites in pre-school children from homes being attended as part of a state programme in Armenia, Colombia

Objective Determining the prevalence of giardiasis and intestinal parasitism in pre-school children from the Instituto Colombiano de Bienestar Familiar (ICBF).

Material and Methods Three serial faecal samples were collected from 328 children following informed consent from their legal guardians. Microscopic and macroscopic tests were made using $1 \%$ Lugol, 0,83 \% saline solution, saline-eosin solution and Kato Katz and Ritchie's test as a confirming test. Positive samples were preserved in saline formol solution.

Results Pathogen parasites had the following prevalence frequencies: 2,4 $\%$ for Ascaris lumbricoides, $0,6 \%$ for Hymenolepsis nana, 2,1 \% for Trichuris trichura, 6,1\% for Blastocystis hominis and $13 \%$ for Giardia lamblia.

Conclusions The results of this work showed low helminth frequency compared to that for protozoa; control strategies using single doses of Albendazol are therefore not useful. The most prevalent pathogen parasite was Giardia lamblia in children from the ICBF in Armenia. Periodic surveillance of parasites in children is thereby recommended. The prevalence of Giardia lamblia was not statistically related to the children's nutritional state. Most Giardia-infected children were asymptomatic.

Key Words: Giardiasis, prevalence, child nutrition, child, pre-school parasite (source: MeSH, NLM). incidencia en regiones tropicales y subtropicales (1). Está demostrado 
que este parásito es el más frecuente en niños preescolares, persistiendo por mas tiempo y con más intensidad la infección que otros parasitos intestinales y causando efectos adversos tanto en el crecimiento y desarrollo, como en el aprendizaje (2-4). Su modo de infección se puede dar principalmente por aguas no tratadas o mal desinfectadas, ya que este parásito sólo es eliminado utilizando una concentración de aproximadamente $8 \mathrm{mg} /$ litro de cloro (los quistes de Giardia sobreviven en el ambiente, particularmente en medios acuáticos). También su infección se puede dar de persona a persona en grupos con deficiente higiene fecal-oral, como ocurre en niños que asisten a guarderías, lo cual es más común de lo que se creía (5). Los focos endémicos presentes en las guarderías constituyen un problema importante, ya que los niños infectados transmiten Giardia a los padres y a otros miembros de la familia y pueden contribuir a mantener una alta endemicidad en las comunidades (1,5-7).

En los Estados Unidos y Canadá es actualmente el parásito entérico más prevalente (1). En Colombia la prevalencia es del 12 \% en la población general y del $28 \%$ entre niños de 1 y 4 años (6). Según el Instituto Nacional de Salud, en la Investigación Nacional de Morbilidad realizada en 1 980, se estableció que el 81,8 \% de las personas en el país se encontraban parasitadas, de éstos el $63 \%$ con parásitos patógenos y $18 \%$ con parásitos no patógenos. Para la zona central, que incluye el departamento del Quindío, en 1980 se reportó una prevalencia de Giardia lamblia del 13,3 \% (6) y en el año 2000 en niños de asentamientos temporales post-terremoto se encontró $60,4 \%$ (8) pero se desconoce la frecuencia actual de parasitismo intestinal en la población de niños de guarderías por fuera de estos asentamientos temporales, y su relación con el estado nutricional. La desnutrición parece ser importante en la población menor de 12 años del departamento del Quindío, según aparece en las estadísticas obtenidas a través del Sistema de Vigilancia Alimentaria y Nutricional -SISVAN- realizado por la subdirección de salud publica del Instituto Seccional de Salud del Quindío (9).

Teniendo en cuenta lo planteado anteriormente, el presente estudio pretende conocer las prevalencias de los diferentes parásitos intestinales, especialmente de Giardia lamblia, en los niños que acuden a los hogares del Instituto Colombiano de Bienestar Familiar (ICBF) del municipio de Armenia y su relación con el estado nutricional. Se espera que los resultados obtenidos permitan obtener un mejor conocimiento sobre la epidemiología del parasitismo intestinal, dados los cambios reportados recientemente en las frecuencias de helmintos y protozoos como consecuencia del aumento en población urbana y con directas implicaciones en las estrategias de desparasitación en pre-escolares. 


\section{MATERIALES Y MÉTODOS}

Población estudiada

Se realizó un estudio de tipo descriptivo de corte transversal, en niños en edad preescolar del municipio de Armenia. Se tomó como universo el total de preescolares (1 a 7 años) que asisten a los 309 hogares comunitarios del Instituto Colombiano de Bienestar Familiar (ICBF) de la Ciudad de Armenia $(\mathrm{N}=3708)$. Con una frecuencia esperada de $15 \%$ de parasitismo y una peor aceptada de 10\% (según estudio de Botero 2002 en Medellín, referencia 7) y que reflejen una confianza del $99 \%$ y un error máximo del $1 \%$, el tamaño de muestra obtenido fue de 310 niños. Se seleccionaron al azar los hogares visitados (35 hogares), uno por cada sector de la ciudad, y en cada uno se estudió el total de niños que asistían al hogar (12 niños). Del estudio se excluyeron los niños con tratamiento parasitario realizado por lo menos 6 meses antes del estudio, la población de preescolares no permanente y los niños mayores de 7 años de edad.

\section{Encuesta y aspectos éticos}

Luego de una charla informativa sobre parasitismo intestinal a los padres o tutores y a las madres comunitarias responsables de cada uno de los hogares, se realizó una encuesta epidemiológica para cada niño. Para esta encuesta se dio información escrita sobre el proyecto a cada responsable y se les invitó a participar de forma voluntaria en el estudio. Se les explicaron los procedimientos a realizar así como los beneficios, en concordancia con la declaración de Helsinki. Se siguieron las normas éticas para trabajos en la comunidad estipuladas en la resolución 008430 de 1993 del Ministerio de Salud Pública de Colombia. Una vez obtenido el consentimiento informado se tomó la muestra de heces y se llenó la encuesta. Si las muestras daban resultados positivos los investigadores suministraron el tratamiento específico de acuerdo al parasito detectado (si helmintos, Albendazol en dosis única de $400 \mathrm{mg}$ o si protozoos, Tinidazol $60 \mathrm{mg} / \mathrm{kg}$ ).

\section{Procesamiento de la muestra y exámenes de laboratorio}

Se tomaron tres muestras seriadas de materia fecal en dias consecutivos, a cada niño. La toma de tres muestras seriadas tiene una sensibilidad del $83 \%$ (10). Las muestras se colectaron en un recipiente estéril, los cuales fueron marcados con el nombre del niño, el centro educativo y la fecha de colección. Las muestras se conservaron refrigeradas hasta su procesamiento. A cada muestra se le hizo un análisis por examen directo con lugol al $1 \%$, 
solución salina al 0,83 \%, solución salina-eosina y Kato-Katz. Adicionalmente a cada muestra se le realizó la técnica de concentración con sulfato de zinc (tecnica de Ritchie) y una coloración con hematoxilina férrica.

\section{Estudio Nutricional}

Para el análisis del estado nutricional se utilizaron las tablas de seguimiento de peso y talla que llevan las madres comunitarias y que se realiza a cada niño de manera trimestral. Se tomó el último dato registrado al momento del muestreo. Se calcularon los indicadores: peso para la edad (P/E), talla para la edad (T/E) y peso para la talla (P/T). Para cada uno de ellos se diagnosticó la situación nutricional de la población estudiada. Se utilizó el sistema de distribución percentilar de la NCHS (National Center For Health Statistic), con una normal de \pm 2 desviaciones éstandar, como datos internacionales de referencia aceptadas en nuestro medio y determinadas por la Organización Mundial de la Salud (11).

\section{Análisis estadístico}

Los datos obtenidos en la aplicación de las encuestas y en el procesamiento de las muestras fueron organizados en tablas electrónicas en el programa Excel (Microsoft), luego se relacionaron las variables frente a los resultados obtenidos y se realizó un análisis estadístico. El análisis estadístico se realizó con el Statcalc del paquete estadístico EpiInfo versión 6 (CDC, 2001).

\section{RESULTADOS}

De un total de 35 hogares seleccionados del ICBF del municipio de Armenia, se estudiaron 328 niños entre 1 y 7 años de edad, de ellos 165 (50,3 \%) fueron niñas y 163 (49,6 \%) fueron niños. Del total de niños evaluados, 155 $(47,2 \%)$ presentaron algún tipo de parásito y 56 (17,2 \%) tuvieron más de una especie de parásito presente en materia fecal (Tabla 1). Entre los parásitos con importancia medica, el de mayor frecuencia fue Giardia lamblia en forma de quiste $(12,8 \%)$. Se encontró también una alta frecuencia de levaduras (28,9\%). Entre los parásitos no patógenos el de mayor frecuencia fue Entamoeba coli (15,5\%).

En la Tabla 2 se muestran los resultados del análisis de asociación entre variables epidemiológicas y los resultados de laboratorio obtenidos. La totalidad de los hogares del ICBF analizados contaban con suministro de agua por acueducto. Giardia estuvo asociada a la presencia de moco en heces, con 
una alta significancía estadística $(\mathrm{p}=0,0005)$. Así mismo se encontró una asociación entre Blastocystis hominis y presencia de congestión nasal de manera estadísticamente significativa $(p=0,02)$. Se realizó un análisis de asociación de Giardia y presencia de otros parásitos. No se encontró asociación significativa estadísticamente con ningún otro parásito, pero si se halló que los niños con levaduras tenían menos presencia de Giardia: solo el 3 \% de los niños con levaduras tenían Giardia mientras que el $16 \%$ de los que no tenían levaduras tenían Giardia, la diferencia fue estadísticamente significativa $(\mathrm{p}=0,002)$. Los mayores porcentajes de prevalencia de Giardia lamblia se registraron entre los niños de 2 y 4 años.

Tabla 1. Prevalencia de parásitos intestinales en 328 niños de 1 a 7 años de 35 hogares de ICBF de la ciudad de Armenia. 2003- 2004

\begin{tabular}{ccc}
\hline Parásito & Frecuencias & $\%$ \\
\hline Levaduras & 93 & 28,9 \\
Entamoeba coli & 51 & 15,5 \\
Endolimax nana & 43 & 13,1 \\
Giardia lamblia* & 42 & 12,8 \\
\hline Comp. E. histolytica/dispar & 30 & 9,1 \\
\hline Blastocystis hominis* & 20 & 6,1 \\
\hline lodamoeba butschlii & 20 & 6,1 \\
\hline Ascaris lumbricoides* & 8 & 2,4 \\
\hline Trichuris trichura* & 7 & 2,1 \\
\hline Hymenolepis nana* & 2 & 0,6 \\
\hline * Parásito patógeno & & \\
\hline
\end{tabular}

*= Parásito patógeno 
Tabla 2. Relación de síntomas o datos de laboratorio en coprológicos con Giardia lamblia y Blastocystis hominis en 328 niños de 1 a 7 años de 35 hogares del ICBF de la ciudad de Armenia 2003-2004

\begin{tabular}{|c|c|c|c|c|c|c|c|c|}
\hline \multirow{2}{*}{ Síntomas } & \multicolumn{4}{|c|}{ Giardia lamblia } & \multicolumn{4}{|c|}{ Blastocystis hominis } \\
\hline & \# & OR & IC $95 \%$ & $\mathrm{p}$ & \# & OR & IC 95\% & $\mathrm{p}$ \\
\hline $\begin{array}{c}\text { Congestión } \\
\text { nasal }\end{array}$ & 23 & 0,6 & $0,3-1,2$ & 0,24 & 18 & 5,4 & $1,2-23,8$ & 0,02 * \\
\hline $\begin{array}{c}\text { Dolor } \\
\text { abdominal }\end{array}$ & 11 & 0,5 & $0,2-1,1$ & 0,17 & 7 & 0,9 & $0,3-2,3$ & 0,95 \\
\hline Diarrea & 4 & 0,5 & $0,1-1,5$ & 0,35 & 4 & 1,3 & $0,4-4,3$ & 0,8 \\
\hline Fiebre & 17 & 1,4 & $0,7-2,7$ & 0,38 & 8 & 1,3 & $0,5-0,43$ & 0,6 \\
\hline $\begin{array}{l}\text { Moco en } \\
\text { heces }\end{array}$ & 18 & 3,3 & $1,7-6,6$ & 0,0005 * & 3 & 0,63 & $0,18-2,23$ & 0,66 \\
\hline $\begin{array}{l}\text { Sangre en } \\
\text { heces }\end{array}$ & 1 & 3,4 & 0,3-39 & 0,84 & 0 & 0,0 & $0,0-0,0$ & 0,44 \\
\hline Tos & 27 & 1,1 & $0,5-2,2$ & 0,82 & 13 & 1,16 & $0,4-3,0$ & 0,9 \\
\hline
\end{tabular}

De los 328 niños, se escogieron 203 niños (61,8 \%) de las guarderías donde se encontró Giardia, para realizar el análisis de asociación con estado nutricional (Tabla 3).

Tabla 3. Relación del análisis nutricional con la prevalencia de Giardia lamblia en los niños de 1 a 7 años de 35 hogares del Instituto Colombiano de Bienestar Familiar ICBF de la ciudad de Armenia. 2003-2004

\begin{tabular}{ccccc}
\hline Variable nutricional & $\begin{array}{c}\text { Niños con desnutrición para } \\
\text { cada variable y con quistes de } \\
\text { Giardia en el coprológico }\end{array}$ & OR & IC $95 \%$ & $P$ \\
P/E & 8 & 1,2 & $0,46-3,16$ & 0,82 \\
T/E & 11 & 1,7 & $0,7-4,2$ & 0,24 \\
P/T & 7 & 1,1 & $0,4-3,0$ & 0,96 \\
\hline Variables nutricionales: peso para la edad (P/E); talla para la edad (T/E); peso para la talla
\end{tabular}
p=Probabilidad; I.C=Intervalo de confianza; O.R=Razón de probabilidades

De éstos, 99 (48,7 \%) fueron niños y 104 (51,2 \%) niñas. En estos 203 niños, 42 (20,6 \%) presentaron Giardia. La prevalencia de Giardia no se relacionó de manera estadísticamente significativa con el estado nutricional de los niños de los hogares del ICBF. El porcentaje de desnutridos crónicos, es decir, para la variable T/E, y que fueron positivos para Giardia fue de $26 \%$, mientras que en los niños sin desnutrición se encontró Giardia en el $16 \%$. En general se encontró mayor porcentaje de desnutrición para P/T o T/E o P/E, en los positivos para Giardia, sobre todo fue mayor en T/E, sin embargo las diferencias no fueron estadísticamente significativas con respecto a los niños sin Giardia. 
Se hizo un análisis sobre el rendimiento del seriado para Giardia en el examen directo y no se encontraron diferencias diagnósticas entre los niños que tuvieron una, dos o tres muestras. De 328 niños, 140 tuvieron solo una muestra, de los cuales 21 fueron positivos para Giardia (15 \%). En 136 niños se examinaron dos muestras con un lapso de tiempo de 48 horas aproximadamente y en este grupo 18 fueron positivos para Giardia (13 \%). En 52 niños se examinaron tres muestras con intervalos de tiempo de 36 horas aproximadamente y se encontraron 3 muestras positivas para Giardia (5\%). Solo un niño entre quienes se examinaron sus tres muestras, fue negativo en su primera muestra y positivo para Giardia en la tercera muestra.

\section{DISCUSIÓN}

El parasitismo intestinal constituye uno de los principales problemas de salud pública en países tropicales y en vía de desarrollo; sin embargo este problema ha sido relegado a un segundo plano seguramente por su bajo índice de mortalidad y a que sus efectos son larvados y a largo plazo (4). En Colombia según la Investigación Nacional de Morbilidad realizada entre 1965 y 1980, se reportó el comportamiento de los parásitos patógenos de la siguiente manera: Ascaris lumbricoides, en 1965 se encontraba en un 54 \% y disminuyó en 1980 a un $34 \%$. Trichuris trichiura de un 50 \% reportado en 1 965, en 1980 disminuyó a un 37 \%. El complejo Entamoeba histolytica/ dispar, en 1965 se reportó con una prevalencia de 24 \% y disminuyó al 12 \% en 1980. Giardia lamblia, al contrario de los parásitos anteriormente nombrados, se comportó de manera ascendente en el tiempo, aumentando su prevalencia de 9,4\% reportada en 1965 a un 21,4 \% en 1980 (6).

En este trabajo se evaluó la prevalencia de parásitos intestinales en una muestra de 328 niños entre 1 y 7 años de edad, pertenecientes a 35 hogares del ICBF del municipio de Armenia, Quindío. En este estudio con lo que respecta a helmintos y cestodos se encontró una prevalencia muy baja de Ascaris lumbricoides (2,4\%), Trichuris trichiura (2,1\%) o de Hymenolepis nana $(0,6 \%)$ con respecto a lo reportado por el Instituto Nacional de Salud en el año 2000. En este estudio nacional las frecuencias para la zona central para T. trichiura fueron de 35,3 \% y para A. lumbricoides de 36,8 \% (6). Estos porcentajes son bajos también con relación a otros estudios realizados en otras regiones de Colombia $(7,13,14)$.

En contraste con los helmintos, se encontró una prevalencia de Giardia lamblia de 12,8 \%. Estos resultados son similares a los reportados por la encuesta nacional de morbilidad, donde se reporta una prevalencia para la zona central de 10,5 \% en 1965 y 13,3 \% en 1980 (6) pero que es baja con rela- 
ción a lo reportado por Lora en el 2001 (8), donde se encontró una prevalencia de 60,4 \% de quistes de Giardia en 217 niños de asentamientos temporales post-terremoto en la ciudad de Armenia. En el estudio post-terremoto, la alta prevalencia se relacionó con el uso de sanitarios comunitarios, las malas condiciones higiénicas del asentamiento y a la utilización de agua de acueducto, pero no de agua de tanque que tenia cloración adicional. En el presente estudio no se pudo comparar entre diferentes fuentes de agua debido a que todos los hogares contaban con suministro de agua de acueducto.

De acuerdo a la presencia o no de síntomas o manifestaciones clínicas, la prevalencia de Giardia lamblia no presentó ninguna asociación estadísticamente significativa para: congestión nasal, fiebre, dolor abdominal, diarrea, o pintas de sangre en las heces. Estos resultados corroboran que la infección por este parásito tiene mayor incidencia en pacientes asintomáticos y por consiguiente se hace más difícil su diagnóstico sin el examen directo de laboratorio (1). En otros estudios, se ha encontrado que el $80 \%$ de los niños de zonas endémicas pobres en Colombia que presentaron quistes de Giardia al examen directo no manifestaron ninguna sintomatología (7). Aunque la presencia de Giardia no se asoció con diarrea, si se encontró asociación, de manera significativa con la presencia de moco en heces, un resultado similar al obtenido previamente en el estudio de Lora et al (8). Se sabe que Giardia crea un efecto de barrera mecánica sobre el intestino delgado, principalmente en el duodeno y yeyuno, llevando a una inflamación intestinal por lesión de las células epiteliales especialmente en las microvellosidades, produciendo un síndrome de malabsorción y en ocasiones ulceración de la mucosa lo cual puede explicar el aumento en las secreciones de moco en el intestino (1).

La prevalencia de levaduras reportada en este estudio (28,9 \%) es la más alta con respecto a las prevalencias encontradas por otros autores. En Vietnam se reportó una prevalencia del $6 \%$ la cual fue asociada a brotes de diarrea (15). Ballal en 1996 (16) reportó en la India una prevalencia de 3,98 \% asociada también a manifestaciones diarreicas y en Arabia se reportó una prevalencia de $0,5 \%$ de levaduras asociadas a diarrea (17). De otro lado hubo menos giardiasis en los niños con levaduras de manera estadísticamente significativa $(\mathrm{p}=0,002)$ lo que posiblemente podría explicarse por una competición por el nicho biológico intestinal.

La variable congestión nasal se asoció de manera significativa con la presencia de Blastocystis hominis $(\mathrm{p}=0,02)$. Una posible explicación a este hallazgo, es la respuesta anormal del sistema inmune frente a estímulos procedentes del parásito y que podrían llevar a una reacción alérgica manifes- 
tada en este caso por congestión nasal. Blastocystis hominis ya se relacionó antes con reacciones alérgicas cutáneas (18).

Respecto al rendimiento del seriado en el examen directo para Giardia, no se mejoró la sensibilidad de la prueba pesar de aumentar el número de exámenes directos. El no aumento del rendimiento del seriado se pudo deber a que las muestras fueron tomadas en días consecutivos y a la deserción que hubo por parte de los acudientes de los niños para completar el número de muestras que se requería para un mejor diagnóstico. Este procedimiento se recomienda según estudios previos, ya que una prueba seriada, es decir, el examen de tres muestras de días no consecutivos, mejora su sensibilidad de un $50 \%$ al tomar una sola muestra a un $90 \%$ con el seriado en días no consecutivos, ya que la eliminación de quistes y trofozoitos es intermitente (10).

En el análisis nutricional no se encontró ninguna asociación estadísticamente significativa entre la presencia de Giardia y desnutrición. Estos resultados fueron similares a los reportados en Medellín (7) y Bogotá (20), confirmando que esta infección es la mayoría de veces asintomática. Es posible que Giardia tenga un efecto larvado de malabsorcion (evidenciado por la presencia de moco en heces) que afecta la incorporación de oligoelementos, pero no de otros nutrientes, lo que explique que no lleve a desnutrición pero si afectar el desarrollo cognitivo, por ejemplo (2). En comparación con el estudio realizado por Cortés donde la desnutrición crónica fue del 45,8 \%, los estados de desnutrición crónica para el total de la población analizada en el presente estudio fueron inferiores (19\%). Este valor resulta al mismo tiempo bajo con relación a los reportados por el Plan Departamental de Alimentación y Nutrición Quindío 2003-2006 (9), donde los promedios de desnutrición crónica para el departamento y el municipio de Armenia en el año 2003 fueron de 41,7 \% y 31,6 \% respectivamente, del mismo modo se encuentran bajos los valores de desnutrición aguda en esta población (15 \%) con respecto a los reportados por el Plan Departamental de Alimentación y Nutrición Quindío, los cuales fueron para el año 2003 en el departamento del Quindío y el municipio de Armenia de 21,4 \% y 31,4 \%. Sin embargo, el valor para considerar desnutrición en estos estudios previos no fue especificado y en algunas cifras que son presentadas en reuniones de la secretaría de salud de Armenia, se presentan valores de desnutrición basadas en el promedio mas una desviación estándar, lo cual obviamente duplicaría las estimaciones de desnutrición. Los criterios a nivel internacional para determinar desnutrición, se basan en promedios menos dos desviaciones estándar, por ello el presente estudio se basó en esta última definición, con el fin de evitar sobreestimaciones de estados de desnutrición. 
En conclusión, se confirmó que el parásito patógeno más prevalente es Giardia lamblia en los niños de los hogares infantiles del ICBF de Armenia. A partir de estos resultados se debe replantear la manera en que se están realizando las desparasitaciones masivas en esta población de preescolares. Estas campañas se realizan con dosis unicas de albendazol, el cual es un antihelmíntico que no es eficaz en dosis única para Giardia. En esta condición de prevalencia lo recomendado por la OMS, cuando existen prevalencias por debajo del $50 \%$, es el tamizaje inicial (pruebas parasito lógicas en coprológicos) y el tratamiento selectivo de los niños que presenten parásitos (20). Dada su importancia como parásito intestinal más prevalente se hace tambien necesario de una parte, determinar el tipo de cepa de Giardia lamblia que está infectando a la población y definir si existe transmisión por perros o gatos y de otro lado, implementar una vigilancia del agua tratada antes de suministrarse a la población con el metodo EPA 1623, tal como se estableció en un estudio anterior (8) •

Agradecimientos. Al Instituto de Bienestar Familiar, Seccional Quindío, por permitir la realización de este estudio.

\section{REFERENCIAS}

1. Mandell G, Benett J, Dolin R. Enfermedades Infecciosas, Principios y Practica 5 ed. Mexico: Ed. Panamericana. 2002; 1:3491.

2. Berkman OS, Lescano AG, Gilman RH, López SL, Black MM. Effects of stunting, diarrhoeal disease and parasitic infection during infancy on cognition in late childhood: a follow up study. Lancet 2002; 16: 564-571.

3. Chan MS. The global burden of intestinal nematode infections. Fifty years on. Parasitol Today 1997; 113(11): 438-43.

4. Savioli L, Bundy D, Tomkins A. Intestinal infections: a soluble public health problem. Trans R Soc Trop Med Hyg 1992; 86: 353-4.

5. Mendoza D, Nuñez F, Escobedo A, Pelayo L, Fernández M, Torres D, Cordoví R. Parasitosis intestinales en 4 círculos infantiles de San Miguel del Padrón, Ciudad de La Habana, 1998. Rev Cubana Med Trop 2001; 53(3): 189-93.

6. Corredor A, Arciniegas E. Parasitismo Intestinal. Instituto Nacional de Salud. Santafé de Bogotá. 2000.

7. Botero J, Castaño A, Montoya M, Hurtado M, Ocampo N, Agudelo G, et al. Anemia por deficiencia de hierro y su asociación con los parásitos intestinales, en escolares y adolescentes matriculados en instituciones oficiales y privadas de Medellín, 1997-1998. Acta Med Col 2002; 27 (1): 7-14.

8. Lora-Suarez F, Marin-Vasquez C, Loango N, Gallego M, Torres E, Gonzalez M, Castano-Osorio J, Gomez-Marin J. Giardiasis in children living in postearthquake camps from Armenia (Colombia). BMC Public Health. 2002; [Internet] Disponible en: http://www.biomedcentral.com/content/pdf/14712458-2-5.pdf. Consultado Agosto de 2005. 
9. Gobernación del Quindío. Comité Técnico Departamental de Alimentación y Nutrición, Plan Departamental de Alimentación y Nutrición, Quindío 20032006. El tren de la buena alimentación. 2003.

10. Nicholls S. Diagnosis of Giardia infections in Colombia. Abstract Volume 1. XV International Congress for Tropical Medicine and Malaria Corcas Editores: Santafé de Bogotá 2000; 223

11. Rojas M, Guerrero L. Nutrición normal en el niño "Generalidades y conceptos" México: Ed Panamericana, 1999; 128-134.

12. Puga-Figueroa L. Protozoos y helmintos intestinales en la población preescolar y escolar de la ciudad de Valdivia, Chile. Parasitol al dia 1991; 15: 57-58.

13. Alvarado BE, Vásquez LR. Determinantes sociales, prevalencia y consecuencias sociales del parasitismo intestinal en población lactante en Guapi, costa Pacífica Caucana. Biomédica 2003; 23: 84-85.

14. Ariza YJ, Sánchez CA, González AM, Ayala JF, Peñaranda M, Castro JI, Lizarazo WF. Estado de las parasitosis intestinales en la población escolar de la esmeralda, Arauquita, Colombia en Junio de 2002. Biomédica 2003; 23: 94.

15. Ngan PK, Khanh NG, Tuong CV, Quy PP; Anh DN, Thuy HT. Persistent diarrea in Vietnamese children: a preliminary report. Acta Paediatr 1992; 381: 1246.

16. Ballal M, Shivananda PG. Rotavirus and enteric pathogens in infantile diarrhoea in Manipal, South India. Indian J Pediatr 2002; 69(5): 393-6.

17. Sheikh SM, el-Assouli SM. Prevalence of viral, bacterial and parasitic enteropathogens among young children with acute diarrhea in Jeddah, Saudi Arabia. J Health Popul Nutr 2001; 19(1): 25-30.

18. Giacometti A, Cirioni O, Autonicelli C, D’Amado G, Silvestri C, Del Pictie MS. Prevalence of intestinal parasitosis among individuals with allegic skin diseases. J Parasitol 2003; 89(3): 490-2.

19. Cortés JR, Salamanca L, Sánchez M, Vanegas F, Sierra PA. Parasitismo y estado nutricional en niños preescolares de instituciones de Santafé de Bogotá. Pediatría 1999; 34 (4). [Internet]. Disponible en: http://www.encolombia.com/. Consultado Septiembre de 2005.

20. Botero D. Community treatments for intestinal nematods infection and drugs of choice. Abstracts. Vol 1. XVth International Congress for Tropical Medicine and Malaria. Cartagena 2000; 205. 